\title{
Impact of concomitant use of DMARDs on the persistence with anti-TNF therapies in patients with rheumatoid arthritis: results from the British Society for Rheumatology Biologics Register
}

\author{
Moetaza M Soliman, ${ }^{1}$ Darren M Ashcroft, ${ }^{1}$ Kath D Watson, ${ }^{2}$ Mark Lunt, ${ }^{2}$ \\ Deborah P M Symmons, ${ }^{2}$ Kimme L Hyrich, ${ }^{2}$ on behalf of the British Society for \\ Rheumatology Biologics Register
}

${ }^{1}$ School of Pharmacy and Pharmaceutical Sciences, The University of Manchester, Manchester, UK

${ }^{2}$ Arthritis Research UK Epidemiology Unit, The University of Manchester, Manchester, UK

\section{Correspondence to} Dr Kimme L Hyrich, Arthritis Research UK Epidemiology Unit, Manchester Academic Health Sciences Centre, The University of Manchester, Oxford Road, Manchester M13 9PT, UK; Kimme.hyrich@manchester. ac.uk

Accepted 1 November 2010 Published Online First 17 February 2011

\begin{abstract}
Objective To evaluate the effect of different concomitant disease modifying antirheumatic drugs (DMARDs) on the persistence with antitumour necrosis factor (anti-TNF) therapies in patients with rheumatoid arthritis (RA).

Method This analysis included 10396 patients with $\mathrm{RA}$ registered with the British Society for Rheumatology Biologics Register, a prospective observational cohort study, who were starting their first anti-TNF therapy and were receiving one of the following DMARD treatments at baseline: no DMARD $(n=3339)$, methotrexate (MTX) ( $n=4418)$, leflunomide (LEF) $(n=610)$, sulfasalazine (SSZ) $(n=308), M T X+S S Z$ $(n=902), M T X+$ hydroxychloroquine (HCO) $(n=401)$ or MTX+SSZ+HCO $(n=418)$. Kaplan-Meier survival analysis was used to study the persistence with antiTNF therapy in each DMARD subgroup up to 5 years. Multivariate Cox proportional hazard models, stratified by anti-TNF used and start year and adjusted for a number of potential confounders, were used to compare treatment persistence overall and according to the reason for discontinuation between each of the DMARD subgroups, using MTX as reference.
\end{abstract}

Results One-year drug survival (95\% Cl) for the first anti-TNF therapy was $71 \%$ (71\% to $72 \%$ ) but this dropped to $42 \%$ ( $41 \%$ to $43 \%$ ) at 5 years. Compared with MTX, patients receiving no DMARD, LEF or SSZ were more likely to discontinue their first anti-TNF therapy while patients receiving MTX in combination with other DMARDs showed better treatment persistence.

Conclusions These results support the continued use of background DMARD combinations which include MTX. Consideration should be given to the discontinuation of LEF and SSZ monotherapy at the time anti-TNF therapies are started, with the possible exception of the SSZ+ETN combination.

\section{INTRODUCTION}

Antitumour necrosis factor (anti-TNF) therapies are now routinely used in the management of rheumatoid arthritis (RA) in patients for whom traditional disease-modifying antirheumatic drugs (DMARDs) have failed. The efficacy of anti-TNF therapies has been evaluated in several randomised controlled trials (RCTs). Recent systematic reviews and meta-analyses have shown their initial efficacy compared with placebo. ${ }^{1-3}$ In addition, a meta-analysis of 13 RCTs has demonstrated better response to three anti-TNF therapies (etanercept (ETN), infliximab (INF) and adalimumab (ADA)) when used in combination with methotrexate (MTX) than when used as monotherapies. ${ }^{1}$ Results from observational studies have also suggested better response to ETN and ADA when prescribed with MTX or with other DMARDs compared with monotherapies. ${ }^{4}$ In clinical practice, although MTX is the DMARD most commonly used with anti-TNF therapies, patients who have a contraindication or intolerance to MTX may still be prescribed anti-TNF therapies either in monotherapy or in combination with other DMARDs. ${ }^{4}$ Further clinical trials and observational studies have suggested the benefits of combined treatment with other concomitant DMARDs including sulfasalazine (SSZ) and hydroxychloroquine (HCQ). ${ }^{5}$ The suggested benefit of combined treatment with leflunomide $(\mathrm{LEF})^{7-11}$ has also been reported, although the results have not been consistent. There has also been a concern about the safety of this latter combination, ${ }^{12}$ with a particular reference to an increased occurrence of dermatological and autoimmune adverse effects. A further proportion of patients will receive anti-TNF therapies in combination with more than one DMARD, which may or may not include MTX. The benefits or safety of continuing these combinations of DMARDs with anti-TNF therapy has not been studied in detail.

Treatment continuation rates (or persistence rates) are an effective tool to measure both the effectiveness and safety of a treatment. ${ }^{13}$ The effect of concurrent DMARDs, primarily MTX, on anti-TNF persistence in RA has been examined in a number of recent studies, ${ }^{7}{ }^{14-18}$ and the results suggested better persistence with anti-TNF therapies when used with DMARDs. However, a majority of these studies were not able to look at individual DMARDs other than MTX owing to small sample size. Therefore, using data from the large British Society for Rheumatology Biologics Register (BSRBR), this study aimed to compare the effects of different concomitant DMARDs, either alone or in combination, on the persistence with anti-TNF therapies in RA. online under the BMJ Journals unlocked scheme, see http:// ard.bmj.com/info/unlocked.dt 


\section{PATIENTS AND METHODS \\ Patient population}

Patients for this analysis were selected from the BSRBR. ${ }^{19}$ The BSRBR is a national prospective observational cohort study based in the UK. The register was established in 2001 by the British Society for Rheumatology (BSR) with the main aim of assessing the long-term safety of biological treatments in RA. In the UK, anti-TNF treatments for RA are reserved for those patients with a 28-joint count Disease Activity Score $(\mathrm{DAS} 28)^{20}>5.1$ despite previous treatment with at least two standard DMARDs (one of which must include MTX). The BSRBR aimed to recruit 4000 patients starting each of the three anti-TNF therapies (ETN, INF and $\mathrm{ADA}$ ); powered on an ability to detect a doubling in the risk of lymphoma compared with standard DMARD treatments. Owing to differences in the availability of each of these three drugs, recruitment to the study was not constant over time and the 4000 target was subsequently achieved for ETN, INF and ADA in 2005, 2007 and 2008, respectively. Ethical approval for the BSRBR was given by the North West Multi-centre Research Ethics Committee in December 2000. All patients provided written informed consent.

\section{Baseline data}

At the start of the anti-TNF treatment, and following patient consent, the rheumatology consultant was asked to complete a consultant baseline questionnaire that collected data on demographics, DAS28, current and previous DMARD treatments, comorbidities and smoking status. The baseline Health Assessment Questionnaire (HAQ) adapted for use in a UK population ${ }^{21}$ was also collected from the patient.

\section{Follow-up}

Follow-up questionnaires were sent to the consultants every 6 months for 3 years and annually thereafter. Consultant follow-up questionnaires collected data on any changes to antiTNF therapies, including start and stop dates and the reasons for stopping the drug (if the patient stopped treatment). The consultant was asked to assign the reasons for stopping from a prespecified list which included inefficacy, adverse events and 'other' reasons.

\section{Data analysis}

BSRBR data up to June 2009 were used for this analysis. All patients with RA registered with the BSRBR who had started treatment with their first course of anti-TNF therapy (ETN, INF or $\mathrm{ADA}$ ) within 6 months of registration were eligible for inclusion in the analysis and classified according to their concomitant DMARD treatments at baseline. Patients receiving the most common DMARD co-therapies (defined as $>2.5 \%$ of the total cohort) were included in the current analysis. Patients for whom no follow-up information was returned (approximately 2.5\%) were excluded.

The primary outcome was persistence with a first anti-TNF therapy, which was defined as the length of time the patients continued to receive their first anti-TNF therapy. ${ }^{22}$ Patients were censored at treatment stop date, date of death or date of the last follow-up, whichever came first. Temporary stops of $<90$ days (which is common for surgery or certain adverse events-for example, infection), after which the patients restarted the same anti-TNF therapy, were not included and counted as continuous use of the drug. Kaplan-Meier survival curves were used to describe the persistence with anti-TNF therapy. Univariate and multivariate Cox proportional hazard models were used to compare anti-TNF discontinuation rates between each concomitant DMARD treatment group and concomitant treatment with MTX (the reference group). Covariates included demographic characteristics (age (years), gender, presence of comorbidities (none/one/more than one), current smoking (yes/no)), baseline disease characteristics (number of previous DMARDs, disease duration (years), baseline DAS28 and baseline HAQ). The models were also stratified by the year of start of anti-TNF therapy, and the anti-TNF therapy used. The primary analysis included all anti-TNF therapies together. A secondary analysis was performed for each anti-TNF therapy separately. In all cases three models were developed: (1) any stop, (2) stopping for inefficacy and (3) stopping for adverse events. The results are presented

Table 1 Baseline characteristics

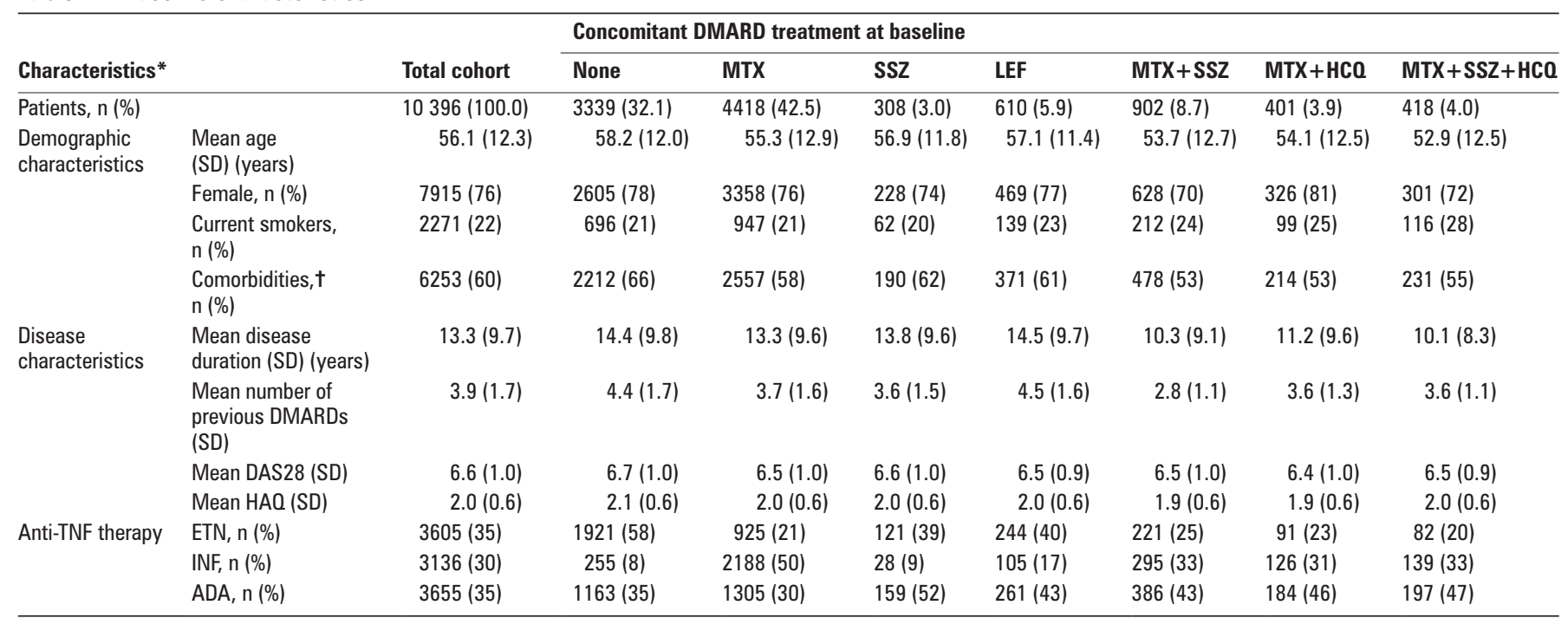

${ }^{*} \mathrm{HAO}$ was available for $95 \%$ of the patients; current smoking and disease duration were available for $99 \%$ of the patients; no missing values were found in the rest of the baseline characteristics.

tComorbidities included one or more of the following: angina, hypertension, myocardial infarction, stroke, epilepsy, asthma, chronic obstructive pulmonary disease, peptic ulcers, liver disease, renal disorder, demyelination, diabetes, hyperthyroidism, depression or a history of tuberculosis or cancer.

ADA, adalimumab; DAS, Disease Activity Score; DMARD, disease-modifying antirheumatic drugs; ETN, etanercept; HAO, Health Assessment Questionnaire; HCQ, hydroxychloroquine; INF, infliximab; LEF, leflunomide; MTX, methotrexate; SSZ, sulfasalazine. 
as hazard ratios and 95\% CIs. STATA 10.1 software was used for all statistical analyses (STATA Corporation, College Station, Texas, USA).

\section{RESULTS}

\section{Baseline characteristics}

By June 2009, 10396 patients registered with the BSRBR met the inclusion criteria for the current analysis (3605 ETN, 3136 INF and $3655 \mathrm{ADA}$ ). Of these, 3339 (32.1\%) were receiving their anti-TNF as monotherapy (ie, no concurrent DMARD at baseline), $4418(42.5 \%)$ in combination with MTX, 610 (5.9\%) with LEF, 308 (3.0\%) with SSZ, 902 (8.7\%) with MTX+SSZ, 401 (3.9\%) with MTX+HCQ and 418 (4.0\%) with $\mathrm{MTX}+\mathrm{SSZ}+\mathrm{HCQ}$.

Baseline characteristics of the entire cohort and stratified by baseline DMARD combination are presented in table 1. Seventysix per cent of patients were female and the mean (SD) age was 56.1 (12.3) years. About one-fifth of the patients were current smokers, while about $60 \%$ of them had other comorbidities in addition to RA. The patients had received a mean (SD) of 3.9 (1.7) DMARDs previously. The patients had a mean (SD) disease duration of 13.3 (9.7) years. The mean (SD) baseline DAS28 was $6.6(1.0)$ and the mean (SD) HAQ score was 2.0 (0.6).

Differences in the baseline characteristics among the patients receiving different baseline DMARD co-therapies were found (table 1). Patients receiving MTX in combination with other DMARDs tended to be younger, have a shorter disease duration and have fewer comorbidities than those receiving either no concomitant DMARDs or DMARD monotherapy. Patients receiving either no DMARDs or LEF with their anti-TNF had received the highest number of previous DMARDs at baseline.

There were also differences in the choice of anti-TNF agent between the different groups. Patients receiving either no DMARD or a DMARD monotherapy other than MTX were less likely to start INF than patients receiving MTX, either alone or in combination.

\section{Treatment persistence}

Over a median of 2.0 years of follow-up ( $\mathrm{IOR}=0.7-4.0$, max 8.1) $50 \%$ of patients discontinued their primary anti-TNF therapy, including $22 \%$ for inefficacy and $21 \%$ for adverse events (table 2). The median overall drug survival was 3.32 years. The overall anti-TNF persistence $(95 \%$ CI) decreased from $71 \%$ $(71 \%$ to $72 \%)$ at year 1 to $42 \%(41 \%$ to $43 \%)$ at year 5 (figure 1 ). Crude treatment persistence of each DMARD combination is shown in figure 2 . The best overall persistence was seen among those patients receiving anti-TNF therapy in combination with MTX plus one or more other DMARD. MTX and SSZ showed similar overall persistence while patients receiving LEF had a persistence profile which was similar to those receiving no concurrent DMARDs.

After adjusting for differences in baseline covariates, compared with MTX, patients receiving MTX in combination with other DMARDs were less likely to discontinue their anti-TNF therapies while patients receiving SSZ or LEF monotherapy, or no concomitant DMARDs were more likely to discontinue their anti-TNF therapies (table 3). Similar results were found when the analysis was limited by the reason for discontinuation (inefficacy or adverse events). A secondary analysis stratified by antiTNF therapy (ETN, INF or ADA) found very similar results to the cohort as a whole with the exception of similar treatment persistence between SSZ and MTX in patients receiving ETN

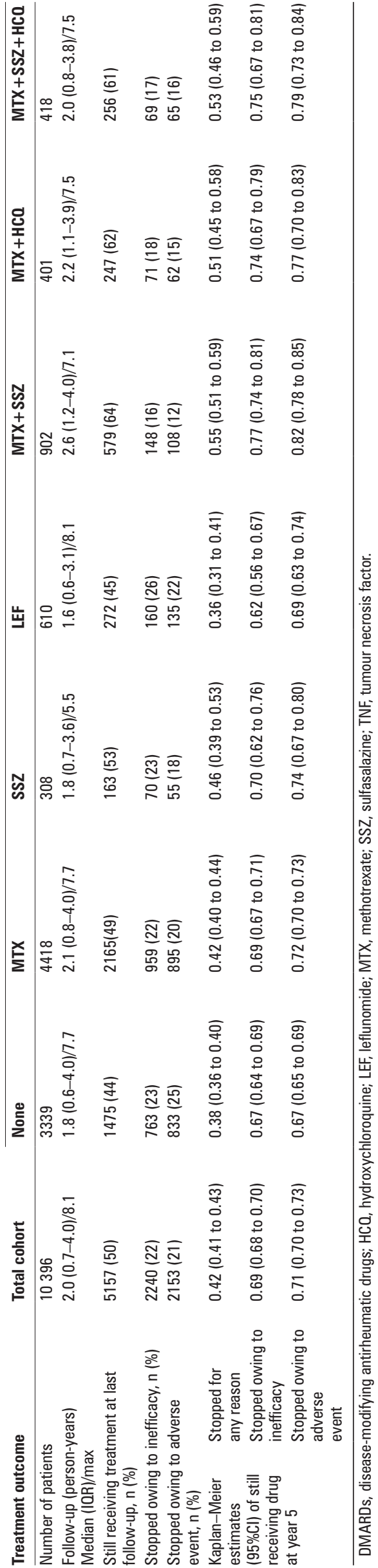


(table 4). However, a test for interaction between SSZ and the anti-TNF therapies was not statistically significant $(p=0.09)$.

\section{DISCUSSION}

This analysis is the first to examine and compare the effects of anti-TNF therapy with a wide range of different DMARD combinations and in particular, the effects of more than one DMARD in combination with anti-TNF. In addition, this study

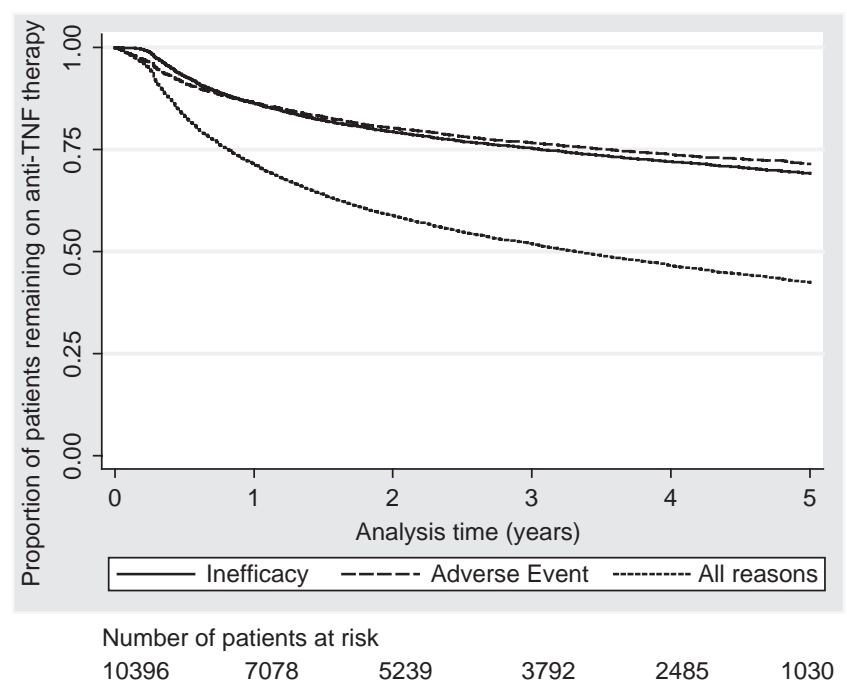

Figure 1 Kaplan-Meier estimates of crude persistence with antitumour necrosis factor (anti-TNF) therapies overall and by reason for discontinuation. presented anti-TNF treatment persistence up to 5 years, at which point $<50 \%$ of patients were still receiving their first anti-TNF therapy.

Our data have confirmed the benefits of combining anti-TNF therapies with MTX. With INF, which is recommended for use with MTX, ${ }^{23}$ the benefit is felt, in part, to be due to the reduction of human antichimeric antibodies. ${ }^{24} 25$ Although the mechanism of benefit is less well understood with other anti-TNF agents, our results are in keeping with the results of both clinical trials and observational data. ${ }^{4} 2627$

One of the strengths of the BSRBR is its large size which has allowed the study of other specific anti-TNF/DMARD combinations. Although the results can only be generalised to patients with high disease activity at start of treatment (DAS28>5.1) despite two failed DMARDs, compared with concurrent MTX, the combination of anti-TNF with either LEF or SSZ resulted in lower treatment persistence, with patients more likely to stop for either inefficacy or adverse events. Previous reports on the benefits or risks of combining anti-TNF therapy with LEF have given mixed results. Two studies have found similar efficacy, whether measured using DAS28, American College of Rheumatology scores or treatment persistence, between anti-TNF in combination with either MTX or LEF. 8 A further Dutch study (n=162), which compared anti-TNF persistence between those receiving LEF and those receiving any other $\operatorname{DMARD}(\mathrm{s})$, also found similar outcomes. ${ }^{28}$ In contrast Strangfeld et a ${ }^{9}$ reported higher discontinuation rates of anti-TNF therapies (especially with INF) ( $n=1769)$ when combined with LEF than when combined with MTX, although this did not reach statistical significance. Other studies, the majority limited to the combination of LEF

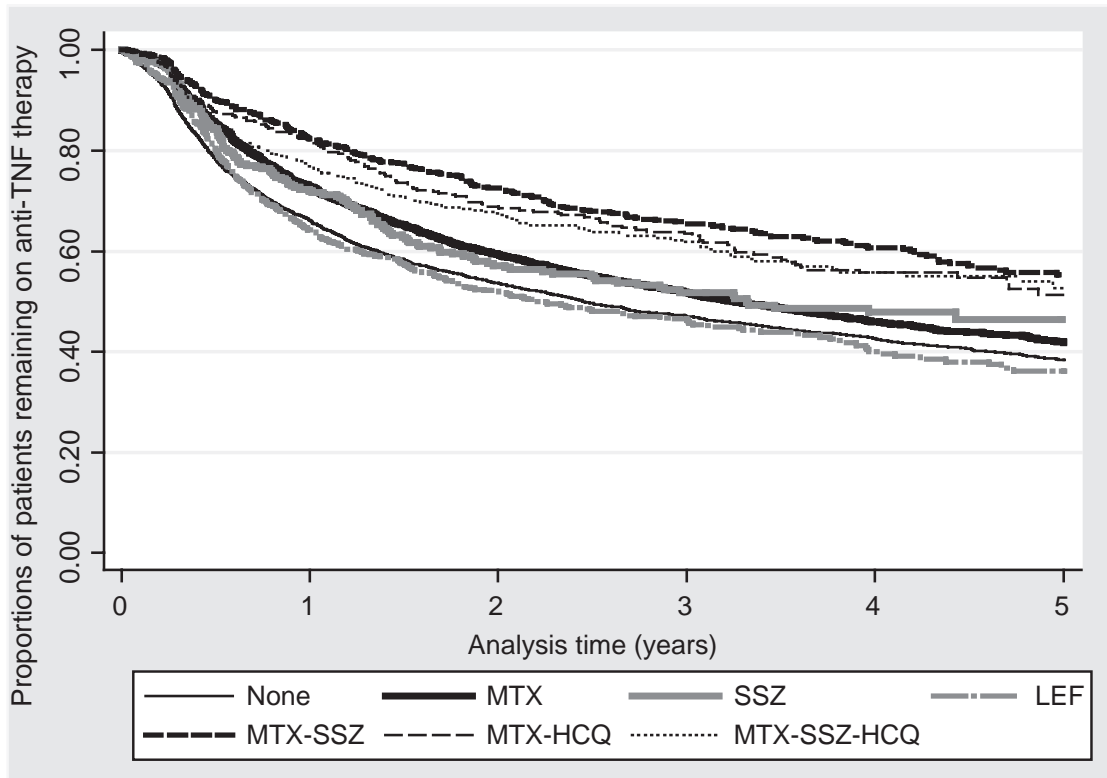

Number of patients at risk

$\begin{array}{lllllll}\text { None } & 3,339 & 2111 & 1580 & 1212 & 848 & 325 \\ \text { MTX } & 4,418 & 3069 & 269 & 1632 & 1060 & 496 \\ \text { SSZ } & 308 & 211 & 144 & 99 & 71 & 18 \\ \text { LEF } & 610 & 379 & 265 & 180 & 99 & 41 \\ \text { MTX-SSZ } & 902 & 706 & 544 & 379 & 242 & 88 \\ \text { MTX-HCQ } & 401 & 311 & 221 & 154 & 89 & 32 \\ \text { MTX-SSZ_HCQ } & 418 & 294 & 219 & 140 & 81 & 32\end{array}$

Figure 2 Kaplan-Meier estimates of crude persistence with anti-tumour necrosis factor (anti-TNF) therapies according to baseline disease-modifying antirheumatic drug(s). HCQ, hydroxychloroquine; LEF, leflunomide; MTX, methotrexate; SSZ, sulfasalazine. 
Table 3 Cox proportional hazard estimates (95\% CI) for anti-TNF therapy discontinuation

\begin{tabular}{|c|c|c|c|c|c|c|}
\hline \multirow{3}{*}{$\begin{array}{l}\text { DMARD co-therapy at } \\
\text { baseline }\end{array}$} & \multicolumn{6}{|c|}{ Hazard ratios $(95 \% \mathrm{Cls})$} \\
\hline & \multicolumn{2}{|l|}{ Overall stopping } & \multicolumn{2}{|c|}{ Stopping owing to inefficacy } & \multicolumn{2}{|c|}{ Stopping owing to adverse events } \\
\hline & Unadjusted & Adjustedt & Unadjusted & Adjustedt & Unadjusted & Adjustedt \\
\hline MTX & Reference & & & & & \\
\hline None & 1.54 (1.43 to 1.65$)$ & $1.40(1.30$ to 1.51$)$ & 1.45 (1.30 to 1.62$)$ & 1.34 (1.20 to 1.51$)$ & 1.69 (1.52 to 1.89$)$ & $1.47(1.30$ to 1.65$)$ \\
\hline SSZ & $1.22(1.03$ to 1.45$)$ & $1.23(1.03$ to 1.47$)$ & 1.34 (1.05 to 1.71$)$ & $1.34(1.04$ to 1.74$)$ & $1.16(0.88$ to 1.52$)$ & $1.21(0.91$ to 1.60$)$ \\
\hline LEF & $1.50(1.34$ to 1.69$)$ & 1.41 (1.25 to 1.59$)$ & 1.64 (1.38 to 1.94 ) & 1.58 (1.32 to 1.88$)$ & $1.47(1.22$ to 1.76$)$ & 1.34 (1.10 to 1.62$)$ \\
\hline $\mathrm{MTX}+\mathrm{SSZ}+\mathrm{HCO}$ & $0.82(0.70$ to 0.96$)$ & $0.80(0.68$ to 0.95$)$ & $0.80(0.62$ to 1.02$)$ & 0.75 (0.58 to 0.96$)$ & $0.83(0.64$ to 1.07$)$ & 0.87 (0.67 to 1.13$)$ \\
\hline p Value* & $<0.01$ & $<0.01$ & $<0.01$ & $<0.01$ & $<0.01$ & $<0.01$ \\
\hline
\end{tabular}

†Adjusted for baseline age, gender, presence of comorbidities, current smoking, number of previous DMARDs, current steroids, disease duration, baseline DAS28 and baseline HAO. All models additionally stratified by start year and anti-TNF agent.

*Test of significance of difference between the different DMARD co-therapies.

DAS, Disease Activity Score; DMARD, disease modifying antirheumatic drugs; HAO, Health Assessment Questionnaire; HCQ, hydroxychloroquine; LEF, leflunomide; MTX, methotrexate; SSZ, sulfasalazine; TNF, tumour necrosis factor.

with INF, have reported acceptable efficacy, although there has been a concern about the safety and longer-term tolerability of anti-TNF when combined with LEF. ${ }^{11} 29-32$ In particular, there has been a concern about dermatological and autoimmune adverse events. A small study from Leeds, UK, reported a high incidence of induction of antinuclear antibodies and doublestranded DNA antibodies in patients receiving INF with LEF. Although there was no control group in this report, there was a high incidence of immune-related adverse events leading to anti-TNF discontinuation. ${ }^{12}$ In the current analysis, combination of LEF with INF was less common than with either ETN or ADA, although a stratified analysis found similar results across all three agents.

Similar results to those for LEF were found with the combination of SSZ with anti-TNF, particularly when SSZ was combined with the monoclonal antibodies, INF and ADA. Less has been published on this combination. A clinical trial found a similar efficacy between ETN monotherapy and ETN+SSZ in SSZ failures. ${ }^{5}$ In contrast, in our study, ETN+SSZ appeared to result in better outcomes than ETN monotherapy, although the difference in persistence between the three anti-TNF therapies did not reach statistical significance.

This is the first study to report on the benefits of combining anti-TNF with multiple DMARDs including MTX. Not only were these patients less likely to stop owing to inefficacy, they were also less likely to experience adverse events leading to drug discontinuation. The biological mechanisms behind this observation remain less clear. One of the limitations of this study is the lack of randomisation. Therefore, in addition to identified differences between the patients receiving different DMARD co-therapies, which were adjusted for in our analysis, there may also have been unmeasured confounders present which may explain in part our findings. One possible unmeasured confounder may be that patients able to tolerate combination DMARDs before starting anti-TNF therapy may be more likely to tolerate new treatments in general. This hypothesis is supported by the higher rate of adverse events observed among patients receiving no concurrent DMARD. We also noted that patients receiving combination DMARDs were more likely to be receiving ADA, which may be related to the timing of when they joined the register, rather than to doctor or patient choice. Owing to recruitment patterns, patients recruited in the later years of the study (when combination DMARD treatment had generally become more widespread) were more likely to be those receiving ADA. These patients also had lower disease duration and therefore, may have had less severe disease overall. Although the results were adjusted for both choice of anti-TNF therapy and year of drug start, these differences in outcome still persisted. With these limitations in mind, the results of this study cannot be interpreted as indicating combination DMARD treatment should be started in patients currently receiving DMARD monotherapy, but as an indication that there is no reason to stop combination DMARDs (at least within the limitations of those combinations analysed) because of concerns about future inefficacy or safety.

Despite the large overall sample size, the size of some groups was still small, limiting statistical power in our secondary analysis stratified by anti-TNF therapy. However, this study does remain the largest of its size and nature, with detailed follow-up now past 5 years for many patients. A further limitation may have existed in the definition of inefficacy and adverse events. The decision to stop anti-TNF therapy and the reason for discontinuation was left to the treating doctor. In some cases, the reasons for stopping may not be completely clear and may fall into more than one category. However, in all cases, we asked for only one reason to be assigned.

Finally, this analysis only took into account which DMARDs patients were taking at baseline and can be seen as the outcomes following the decision to treat with that DMARD or combination, rather than as outcomes following a given period of treatment (an intention-to-treat analysis). Many patients may have either decreased or indeed started new DMARDs after the introduction of anti-TNF based on initial response, which may have also influenced longer-term drug persistence. A future analysis that included the use of these alternative DMARD treatments throughout the period of follow-up may find different results but would need an even larger sample size to account for the multiple possible combinations of treatment.

In conclusion, we have shown that compared with combining anti-TNF with MTX, combining anti-TNF with MTX in combination with one or more other DMARDs (SSZ, HCQ or both) resulted in better long-term treatment persistence. However, combination of anti-TNF with LEF and SSZ (with the exception of ETN+SSZ) resulted in higher rates of discontinuation owing to both inefficacy and safety. As the potential for unmeasured confounding is present in this study, further study is required to understand if longer-term anti-TNF treatment persistence would be improved by simply stopping these DMARDs or whether they should be substituted, where possible, with other DMARDS such as MTX, if tolerated. 


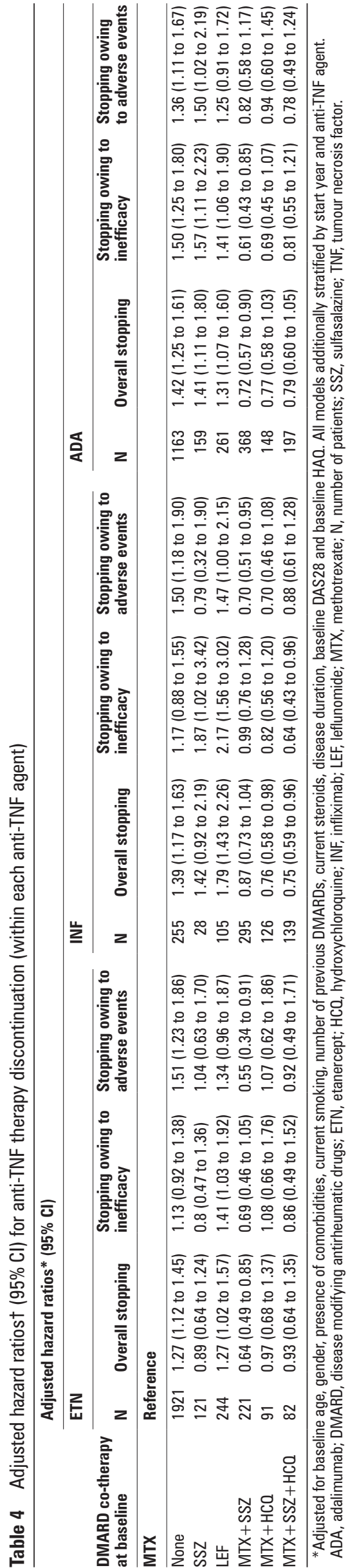

Acknowledgements The authors acknowledge the enthusiastic collaboration of all consultant rheumatologists and their specialist nurses in the UK in providing the data. In addition, we acknowledge the support from Dr lan Griffiths (past) and Professor David Isenberg (current), Chairs of the BSRBR Management Committee, Professor Gabriel Panayi, Professor David G I Scott, Dr Andrew Bamii, Dr Deborah Bax and Dr David L Scott, Presidents of the BSR during the period of data collection, for their active role in enabling the Register to undertake its tasks and to Samantha Peters (CEO of the BSR), Nia Taylor, Mervyn Hogg, Nia Taylor and members of the BSRBR Scientific Steering Committee. We also acknowledge the seminal role of the BSR Clinical Affairs Committee for establishing national biologic guidelines and recommendations for such a Register. Finally we would like to acknowledge the substantial contribution of Andy Tracey, Katie McGrother and Dr Mark Lunt in database design and manipulation and Professor Alan Silman in his prior role as a principal investigator of the BSRBR.

Funding Funding for the BSR Biologics Register was provided by the British Society for Rheumatology.

Competing interests The British Society for Rheumatology (BSR) commissioned the Biologics Register (BSRBR) as a UK-wide national project to investigate the safety of biological agents in routine medical practice. DPMS and KLH are principal investigators on the BSRBR. BSR receives restricted income from UK pharmaceutical companies, presently Abbott Laboratories, Amgen, Roche, Schering Plough and Wyeth Pharmaceuticals. This income finances a wholly separate contract between the BSR and the University of Manchester who provide and run the BSRBR data collection, management and analysis services. The principal investigators and their team have full academic freedom and are able to work independently of pharmaceutical industry influence. All decisions about analyses, interpretation and publication are made autonomously of any industrial contribution. Members of the Manchester team, BSR trustees, committee members and staff complete an annual declaration in relation to conflicts of interest. The PhD work of MMS was supported by the Egyptian Government. The authors declare no other conflicts of interest.

Ethics approval This study was conducted with the approval of the North West Multi-centre Research Ethics Committee.

Provenance and peer review Not commissioned; externally peer reviewed.

\section{REFERENCES}

1. Nixon R, Bansback N, Brennan A. The efficacy of inhibiting tumour necrosis factor alpha and interleukin 1 in patients with rheumatoid arthritis: a meta-analysis and adjusted indirect comparisons. Rheumatology (Oxford) 2007:46:1140-7.

2. Venkateshan SP, Sidhu S, Malhotra S, et al. Efficacy of biologicals in the treatment of rheumatoid arthritis. a meta-analysis. Pharmacology 2009;83:1-9.

3. Wiens A, Correr CJ, Pontarolo R, et al. A systematic review and meta-analysis of the efficacy and safety of etanercept for treating rheumatoid arthritis. Scand J Immunol 2009;70:337-44.

4. Hyrich KL, Symmons DP, Watson KD, et al. Comparison of the response to infliximab or etanercept monotherapy with the response to cotherapy with methotrexate or another disease-modifying antirheumatic drug in patients with rheumatoid arthritis: results from the British Society for Rheumatology Biologics Register. Arthritis Rheum 2006:54:1786-94.

5. Combe B, Codreanu C, Fiocco U, et al. Etanercept and sulfasalazine, alone and combined, in patients with active rheumatoid arthritis despite receiving sulfasalazine: a double-blind comparison. Ann Rheum Dis 2006:65:1357-62.

6. O'Dell JR, Petersen K, Leff R, et al. Etanercept in combination with sulfasalazine, hydroxychloroquine, or gold in the treatment of rheumatoid arthritis. J Rheumatol 2006;33:213-18.

7. Finckh A, Dehler S, Gabay C. The effectiveness of leflunomide as a co-therapy of tumour necrosis factor inhibitors in rheumatoid arthritis: a population-based study. Ann Rheum Dis 2009;68:33-9.

8. De Stefano R, Frati E, Nargi F, et al. Comparison of combination therapies in the treatment of rheumatoid arthritis: leflunomide-anti-TNF-alpha versus methotrexateanti-TNF-alpha. Clin Rheumatol 2010;29:517-24.

9. Strangfeld A, Hierse F, Kekow J, et al. Comparative effectiveness of tumour necrosis factor alpha inhibitors in combination with either methotrexate or leflunomide. Ann Rheum Dis 2009;68:1856-62.

10. Kalden JR, Antoni C, Alvaro-Gracia JM, et al. Use of combination of leflunomide with biological agents in treatment of rheumatoid arthritis. J Rheumatol 2005;32:1620-31.

11. Kalden JR, Nüsslein HG, Wollenhaupt J, et al. Combination treatment with infliximab and leflunomide in patients with active rheumatoid arthritis: safety and efficacy in an open-label clinical trial. Clin Exp Rheumatol 2008;26:834-40.

12. Bingham SJ, Buch MH, Kerr MA, et al. Induction of antinuclear antibodies in patients with rheumatoid arthritis treated with infliximab and leflunomide. Arthritis Rheum 2004;50:4072-3.

13. Geborek $\mathbf{P}$, Crnkic $M$, Petersson IF, et al. Etanercept, infliximab, and leflunomide in established rheumatoid arthritis: clinical experience using a structured follow up programme in southern Sweden. Ann Rheum Dis 2002;61:793-8. 
14. Heiberg MS, Koldingsnes W, Mikkelsen K, et al. The comparative one-year performance of anti-tumor necrosis factor alpha drugs in patients with rheumatoid arthritis, psoriatic arthritis, and ankylosing spondylitis: results from a longitudinal, observational, multicenter study. Arthritis Rheum 2008;59:234-40.

15. Kristensen LE, Saxne T, Nilsson JA, et al. Impact of concomitant DMARD therapy on adherence to treatment with etanercept and infliximab in rheumatoid arthritis. Results from a six-year observational study in southern Sweden. Arthritis Res Ther 2006;8:R174.

16. Ostergaard $\mathbf{M}$, Unkerskov J, Linde L, et al. Low remission rates but long drug survival in rheumatoid arthritis patients treated with infliximab or etanercept: results from the nationwide Danish DANBIO database. Scand J Rheumatol 2007;36:151-4.

17. Tang B, Rahman M, Waters HC, et al. Treatment persistence with adalimumab, etanercept, or infliximab in combination with methotrexate and the effects on health care costs in patients with rheumatoid arthritis. Clin Ther 2008;30:1375-84.

18. Zink A, Listing J, Kary S, et al. Treatment continuation in patients receiving biological agents or conventional DMARD therapy. Ann Rheum Dis 2005;64:1274-9.

19. Silman A, Symmons D, Scott DG, et al. British Society for Rheumatology Biologics Register. Ann Rheum Dis 2003;62(Suppl 2):ii28-ii29.

20. Prevoo ML, van 't Hof MA, Kuper HH, et al. Modified disease activity scores that include twenty-eight-joint counts. Development and validation in a prospective longitudinal study of patients with rheumatoid arthritis. Arthritis Rheum 1995;38:44-8.

21. Bruce B, Fries JF. The Health Assessment Questionnaire (HAO). Clin Exp Rheumatol 2005;23(5 Suppl 39):S14-8.

22. Saad AA, Ashcroft DM, Watson KD, et al. Persistence with anti-tumour necrosis factor therapies in patients with psoriatic arthritis: observational study from the British Society of Rheumatology Biologics Register. Arthritis Res Ther 2009;11:R52

23. National Institute for Health and Clinical Effectiveness Technology Appraisal Guidance 130. Adalimumab, etanercept and infliximab for the treatment of rheumatoid arthritis. http://www.nice.org.uk/nicemedia/pdf/TA130guidance.pdf (accessed 3 May 2010).
24. Maini R, St Clair EW, Breedveld F, et al. Infliximab (chimeric anti-tumour necrosis factor alpha monoclonal antibody) versus placebo in rheumatoid arthritis patients receiving concomitant methotrexate: a randomised phase III trial. ATTRACT Study Group. Lancet 1999;354:1932-9.

25. Maini RN, Breedveld FC, Kalden JR, et al. Therapeutic efficacy of multiple intravenous infusions of anti-tumor necrosis factor alpha monoclonal antibody combined with low-dose weekly methotrexate in rheumatoid arthritis. Arthritis Rheum 1998;41:1552-63.

26. Klareskog L, van der Heijde D, de Jager JP, et al. Therapeutic effect of the combination of etanercept and methotrexate compared with each treatment alone in patients with rheumatoid arthritis: double-blind randomised controlled trial. Lancet 2004;363:675-81.

27. Breedveld FC, Weisman MH, Kavanaugh AF, et al. The PREMIER study: A multicenter, randomized, double-blind clinical trial of combination therapy with adalimumab plus methotrexate versus methotrexate alone or adalimumab alone in patients with early, aggressive rheumatoid arthritis who had not had previous methotrexate treatment. Arthritis Rheum 2006;54:26-37.

28. Flendrie $\mathbf{M}$, Creemers $\mathrm{MC}$, Welsing PM, et al. The influence of previous and concomitant leflunomide on the efficacy and safety of infliximab therapy in patients with rheumatoid arthritis; a longitudinal observational study. Rheumatology (Oxford) 2005:44:472-8.

29. Godinho F, Godfrin B, El Mahou S, et al. Safety of leflunomide plus infliximab combination therapy in rheumatoid arthritis. Clin Exp Rheumatol 2004:22:328-30.

30. Hansen KE, Cush J, Singhal A, et al. The safety and efficacy of leflunomide in combination with infliximab in rheumatoid arthritis. Arthritis Rheum 2004;51:228-32.

31. Kiely PD, Johnson DM. Infliximab and leflunomide combination therapy in rheumatoid arthritis: an open-label study. Rheumatology (Oxford) 2002;41:631-7.

32. Alcorn N, Saunders S, Madhok R. Benefit-risk assessment of leflunomide: an appraisal of leflunomide in rheumatoid arthritis 10 years after licensing. Drug Saf 2009:32:1123-34 